\title{
Comonotonic asset prices in arbitrage-free markets
}

\author{
Jan Dhaene* $\quad$ Alexander Kukush ${ }^{\dagger} \quad$ Daniël Linders ${ }^{\ddagger}$
}

January 6, 2019

\begin{abstract}
For an arbitrage-free market with a single underlying asset, we investigate conditions under which the consecutive price levels are comonotonic. Furthermore, for an arbitrage-free market with $n$ assets we investigate the consequences of assuming comonotonicity of the vector containing the price levels of each asset at a single future date $T$. Although being of a theoretical nature, the results of this paper give insight in the reachability of the comonotonic upper bounds for Asian options and for basket options that can be found in Simon et al. (2000), Dhaene et al. (2002b), Hobson et al. (2005) and Chen et al. (2008).
\end{abstract}

\section{Introduction}

Random variables (r.v.'s) are said to be comonotonic if they are all non-decreasing (or nonincreasing) functions of the same random source. As a result, comonotonicity describes a situation where the r.v.'s are moving perfectly together. The concept of comonotonicity has been investigated in detail in the actuarial and financial literature; see e.g. Dhaene et al. (2002a b), Deelstra et al. (2011). Assuming a comonotonic dependence structure between asset prices has proven to be a useful tool to solve several financial applications such as derivative pricing (Deelstra et al. (2004), Deelstra et al. (2008) and Linders \& Stassen (2016)) and dependence measurement; see e.g. Dhaene, Dony, Forys, Linders \& Schoutens (2012), Dhaene, Linders, Schoutens \& Vyncke (2012), Dhaene et al. (2014) and Linders et al. (2012), to name few.

In this paper, we first consider an arbitrage-free market consisting of a risk-free bank account and a single risky asset. Today is time $t=0$ and we consider the random price levels $S\left(t_{1}\right), S\left(t_{2}\right), \ldots, S\left(t_{n}\right)$ at the discrete set of times $0<t_{1}<t_{2}<\ldots<t_{n}$. We derive conditions under which the real world as well as the risk-neutral distribution of

*KU Leuven, Leuven, Belgium. Email: Jan.Dhaene@kuleuven.be

${ }^{\dagger}$ Faculty of Mechanics and Mathematics, Taras Shevchenko National University of Kyiv, Kyiv, Ukraine. Email: alexander_kukush@univ.kiev.ua

${ }^{\ddagger}$ University of Illinois at Urbana-Champaign, Urbana, USA. Email: dlinders@illinois.edu 
the random vector $\left(S\left(t_{1}\right), S\left(t_{2}\right), \ldots, S\left(t_{n}\right)\right)$ can be comonotonic. To be more precise, we prove that for popular continuous models such as the Black \& Scholes model, the comonotonicity property is in contradiction with the no-arbitrage assumption. We also provide examples of stock price models where the price vector $\left(S\left(t_{1}\right), S\left(t_{2}\right), \ldots, S\left(t_{n}\right)\right)$ may be comonotonic, without contradicting the no-arbitrage assumption.

Consider the Asian option with strike $K$ and maturity $T$ whose pay-off at maturity is equal to $\left(\frac{1}{n} \sum_{i=1}^{n} S\left(t_{i}\right)-K\right)_{+}$. Knowledge about the dynamics of the price vector $\left(S\left(t_{1}\right), S\left(t_{2}\right), \ldots, S\left(t_{n}\right)\right)$ is required in order to price this Asian option. Suppose that also vanilla options on the stock $S$ with maturity $t_{i}, i=1,2, \ldots, n$ are traded. Their prices give information about the risk-neutral cdf's of the $S\left(t_{i}\right)$. However, this information is not sufficient to specify the risk-neutral cdf of $\left(S\left(t_{1}\right), S\left(t_{2}\right), \ldots, S\left(t_{n}\right)\right)$. One may search for an upper bound of the price of an Asian option in the class of models which are consistent with the traded vanilla option prices; see e.g. Kerkhof et al. $(\overline{2010})$ and Barrieu \& Scandolo (2013). In case comonotonicity of $\left(S\left(t_{1}\right), S\left(t_{2}\right), \ldots, S\left(t_{n}\right)\right)$ does not violate the no arbitrage assumption, this 'worst case price' can be derived using the prices of traded vanilla options and then coincides with the comonotonic upper bound for the Asian option price which was derived in Simon et al. (2000), Dhaene et al. (2002b) and Albrecher et al. (2005). We show that for continuous stock price models such as the Black \& Scholes model and a variety of Lévy models, a comonotonic stock price vector is not attainable. The comonotonic upper bound for the Asian option will be free of any model risk, but not reachable.

Next, we consider an arbitrage-free market consisting of $n$ traded (dividend or nondividend paying) assets and a risk-free bank account. The price of asset $i$ at time $T$ is denoted by $S_{i}$. We explore situations under which the price vector $\left(S_{1}, S_{2}, \ldots, S_{n}\right)$ is comonotonic and arbitrage-free. In particular, we have a closer look at the multivariate Black \& Scholes model and give examples of comonotonic price vectors. In Simon et al. (2000), Dhaene et al. (2002b) and Chen et al. (2008), the authors show that the situation where the price vector $\left(S_{1}, S_{2}, \ldots, S_{n}\right)$ is comonotonic can be artificially created using vanilla option data.

A basket option written on the stocks $S_{1}(T), S_{2}(T), \ldots, S_{n}(T)$ with strike $K$, maturity $T$ has a pay-off at maturity equal to $\left(\sum_{i=1}^{n} w_{i} S_{i}(T)-K\right)_{+}$, where all $w_{i}>0$. Pricing the basket option requires knowledge about the marginal cdf's and the dependence among the components. Similar to the Asian option case, any misspecification of the dependence structure leads to a wrongly priced basket option and one can try to reduce the modelrisk by determining the basket option price in a 'worst case scenario' among the class of models which are consistent with the observed vanilla option prices. In this paper, we show that in case of a basket option, this worst case scenario is always described by comonotonicity of the stock price vector $\left(S_{1}(T), S_{2}(T), \ldots, S_{n}(T)\right)$. Furthermore, the price of the basket option in this comonotonic scenario is always free of arbitrage and is equal to an upper bound which is free of any model risk.

This paper is structured as follows. In Section 2 we briefly recapitulate the concept of comonotonicity and some of its properties that we will need for proving our main results. In Section 3 we describe the general financial market in which our results hold. In Section 4 we first present results concerning the comonotonicity of the asset prices of a single asset 
at different dates. We consider the concept of comonotonicity of the prices of different assets at a single date in Section 5. Section 6 concludes the paper.

\section{Comonotonic random variables}

In the sequel, the vector $\left(x_{1}, \ldots, x_{n}\right)$ will be denoted by $\underline{x}$. For two vectors $\underline{x}$ and $\underline{y}$ the notation $\underline{x} \leqslant y$ will be used for the componentwise order, which is defined by $x_{i} \leqslant \bar{y}_{i}$ for all $i=1, \ldots, n$. Equalities such as $X=Y$ for the r.v.'s $X$ and $Y$ defined on the probability space $(\Omega, \mathcal{F}, \mathbb{P})$ have to be interpreted as almost sure relationships. Finally, $X \stackrel{d}{=} Y$ means that $X$ and $Y$ are equal in distribution.

Definition 1 A set $A \subseteq \mathbb{R}^{n}$ is said to be comonotonic if for any $\underline{x}$ and $\underline{y}$ in $A$, either $\underline{x} \leqslant \underline{y}$ or $\underline{y} \leqslant \underline{x}$ holds.

Hereafter we restate the definition of the notion of comonotonicity of a random vector, as presented in Kaas et al. (2000).

Definition 2 A random vector $\underline{X}$ is comonotonic if it has a comonotonic support.

The standard notation $F_{X}^{-1}$ will be used to denote the usual inverse of the cumulative distribution function (cdf) $F_{X}$ of the r.v. $X$

$$
F_{X}^{-1}(p)=\inf \left\{x \in \mathbb{R} \mid F_{X}(x) \geq p\right\}, \quad p \in[0,1],
$$

with inf $\varnothing=+\infty$ by convention.

In the following theorem, several characterizations of the comonotonicity of a random vector are presented. For a proof, we refer to Denneberg (1997) or Dhaene et al. (2002a).

Theorem 3 A random vector $\underline{X}=\left(X_{1}, X_{2}, \ldots, X_{n}\right)$ is comonotonic if, and only if, one of the following equivalent conditions holds:

(1) For all $\underline{x}=\left(x_{1}, x_{2}, \ldots, x_{n}\right)$, we have that

$$
F_{\underline{X}}(\underline{x})=\min \left\{F_{X_{1}}\left(x_{1}\right), F_{X_{2}}\left(x_{2}\right), \ldots, F_{X_{n}}\left(x_{n}\right)\right\} .
$$

(2) For $U \sim$ Uniform $(0,1)$, we have that

$$
\underline{X} \stackrel{\mathrm{d}}{=}\left(F_{X_{1}}^{-1}(U), F_{X_{2}}^{-1}(U), \ldots, F_{X_{n}}^{-1}(U)\right) .
$$

(3) There exists a r.v. $Z$ and non-decreasing functions $f_{i}: \mathbb{R} \rightarrow \mathbb{R}, i=1, \ldots, n$, such that

$$
\underline{X} \stackrel{\mathrm{d}}{=}\left(f_{1}(Z), f_{2}(Z), \ldots, f_{n}(Z)\right) \text {. }
$$


(4) There exist continuous and non-decreasing functions $f_{i}: \mathbb{R} \longrightarrow \mathbb{R}$ such that

$$
f_{1}(z)+f_{2}(z)+\ldots+f_{n}(z)=z
$$

for any $z \in \mathbb{R}$ and

$$
\underline{X}=\left(f_{1}(S), f_{2}(S), \ldots, f_{n}(S)\right),
$$

with

$$
S=X_{1}+X_{2}+\ldots+X_{n}
$$

In Expression (6) and below we assume that the equality between the r.v.'s holds $\mathbb{P}$ - a.s. We present some equivalent characterizations for a comonotonic random vector of which at least one component has a continuous cdf. These characterizations will be used to prove our main results. Without loss of generality, we will assume that the first component of the random vector has a continuous cdf.

Theorem 4 Consider a random vector $\underline{X}=\left(X_{1}, X_{2}, . ., X_{n}\right)$ and assume that $X_{1}$ has a continuous cdf. In this case, the following statements are equivalent:

(1) $\underline{X}$ is comonotonic.

(2) There exist non-decreasing functions $f_{i}, i=2,3, \ldots, n$, such that

$$
\underline{X} \stackrel{\mathrm{d}}{=}\left(X_{1}, f_{2}\left(X_{1}\right), f_{3}\left(X_{1}\right), \ldots, f_{n}\left(X_{1}\right)\right) .
$$

(3) There exist non-decreasing functions $f_{i}, i=2,3, \ldots, n$, such that

$$
\underline{X}=\left(X_{1}, f_{2}\left(X_{1}\right), f_{3}\left(X_{1}\right), \ldots, f_{n}\left(X_{1}\right)\right) .
$$

Proof. $(1) \Rightarrow(2)$ : As $F_{X_{1}}$ is continuous, we have that $F_{X_{1}}\left(X_{1}\right)$ is uniformly distributed on the unit interval. Consequently, we also have that

$$
F_{X_{i}}^{-1}\left(F_{X_{1}}\left(X_{1}\right)\right) \stackrel{\mathrm{d}}{=} X_{i}, i=2,3, \ldots, n .
$$

The comonotonic random vector $\left(X_{1}, F_{X_{2}}^{-1}\left(F_{X_{1}}\left(X_{1}\right)\right), F_{X_{3}}^{-1}\left(F_{X_{1}}\left(X_{1}\right)\right), \ldots, F_{X_{n}}^{-1}\left(F_{X_{1}}\left(X_{1}\right)\right)\right)$ has the same marginals as the comonotonic vector $\underline{X}$. This implies that

$$
\underline{X} \stackrel{\mathrm{d}}{=}\left(X_{1}, F_{X_{2}}^{-1}\left(F_{X_{1}}\left(X_{1}\right)\right), F_{X_{3}}^{-1}\left(F_{X_{1}}\left(X_{1}\right)\right), \ldots, F_{X_{n}}^{-1}\left(F_{X_{1}}\left(X_{1}\right)\right)\right) .
$$

We find that $\underline{X} \stackrel{\mathrm{d}}{=}\left(X_{1}, f_{2}\left(X_{1}\right) \ldots, f_{n}\left(X_{1}\right)\right)$ with $f_{i}(x)=F_{X_{i}}^{-1}\left(F_{X_{1}}(x)\right), x \in \mathbb{R}$. (2) $\Rightarrow(1)$ : Trivial.

$(2) \Leftrightarrow(3)$ : The $\Leftarrow$ implication is straightforward. It remains to prove that (2) implies (3). Therefore, assume that $\underline{X} \stackrel{\mathrm{d}}{=}\left(X_{1}, f_{2}\left(X_{1}\right) \ldots, f_{n}\left(X_{1}\right)\right)$. Then for any $i=2,3, \ldots, n$, we find that $\left(X_{1}, X_{i}\right) \stackrel{\mathrm{d}}{=}\left(X_{1}, f_{i}\left(X_{1}\right)\right)$. Hence for any $i=2,3, \ldots, n$, it holds that $\left(f_{i}\left(X_{1}\right), X_{i}\right) \stackrel{\mathrm{d}}{=}$ $\left(f_{i}\left(X_{1}\right), f_{i}\left(X_{1}\right)\right)$, which in turn implies that $X_{i}=f_{i}\left(X_{1}\right)$.

We can conclude that $\underline{X}=\left(X_{1}, f_{2}\left(X_{1}\right) \ldots, f_{n}\left(X_{1}\right)\right)$. 
Theorem 4 is closely related to the concept of strict comonotonicity, defined in Chen et al. (2015). The random vector $\underline{X}$ is strictly comonotonic if it has a support such that for any elements $\underline{x}, \underline{y}$ of this support with $\underline{x} \neq \underline{y}$, it must hold that $\underline{x}<\underline{y}$ or $\underline{x}>\underline{y}$. One can prove that in case all marginals have continuous cdf's, comonotonicity and strict comonotonicity are equivalent. From Theorem 4 we find that, in this situation, comonotonicity of a random vector implies that the outcome of any random variable determines all other outcomes.

Theorem 4 does not necessarily hold in case $X_{1}$ has a discrete cdf. Indeed, consider the random couple $\left(X_{1}, X_{2}\right)$ and suppose that

$$
X_{j+1}=X_{j} Z_{j+1}, \quad j=0,1,
$$

where $X_{0}$ is a given real number and $Z_{1}$ and $Z_{2}$ are i.i.d. r.v.'s which can only take two different values $u>0$ and $d>0$, with $d<u$ and

$$
\mathbb{P}\left[Z_{j}=u\right]=1-\mathbb{P}\left[Z_{j}=d\right]=p,
$$

for some $0<p<1$. The couple $\left(X_{1}, X_{2}\right)$ is comonotonic since it has the following comonotonic support:

$$
\left\{\left(X_{0} d, X_{0} d^{2}\right),\left(X_{0} d, X_{0} d u\right),\left(X_{0} u, X_{0} u d\right),\left(X_{0} u, X_{0} u^{2}\right)\right\} .
$$

As $X_{1}$ has a two-point distribution, while $X_{2}$ has a three-point distribution, it is impossible to find a non-decreasing functions $f$ such that $X_{2}=f\left(X_{1}\right)$. This means that the outcome of $X_{1}$ does not determine unambiguously the realization of $X_{2}$.

\section{The financial market}

Consider the filtered probability space $\left(\Omega, \mathcal{F},\left(\mathcal{F}_{t}\right)_{t \in I}, \mathbb{P}\right)$, where $I$ is either $[0, T]$ or $I=$ $\left\{0, t_{1}, t_{2}, \ldots, t_{N}\right\}$ with $t_{N} \leq T$, to describe a financial market consisting of $n$ traded stocks and a bank account. We assume that the filtered probability space satisfies the usual 'technical' conditions of completeness and right-continuity. Moreover, $\mathcal{F}_{0}$ contains all $\mathbb{P}$-null sets of $\Omega$ and $\mathcal{F}_{T}=\mathcal{F}$. Money can be deposited on or borrowed from the bank at the continuously compounded interest intensity $r \geq 0$, which is assumed to be deterministic and constant over time.

Consider a particular stock and suppose that its current price is denoted by $S(0)>0$, which is assumed to be known. The price of this stock at future dates $t>0$ is described by the adapted stochastic process $\{S(t) \mid t \in I\}$ which is defined on the above mentioned filtered probability space. Our stock price model is very general and accommodates discrete-time as well as continuous-time models for the stock price.

Assume for the moment that the stock price process is continuous, i.e. $I=[0, T]$. The stock is assumed to pay a continuous dividend yield $\nu$, which is assumed to be deterministic and constant over time. Denoting the stochastic value of the (non-discounted) dividends paid in $(0, t)$ by $D(t)$, we have that

$$
\mathrm{d} D(t)=\nu S(t) \mathrm{d} t .
$$


The gains process $\{G(t) \mid 0 \leq t \leq T\}$ is defined by

$$
G(t)=S(t)+\int_{0}^{t} \mathrm{e}^{r(t-u)} \mathrm{d} D(u), \quad t>0,
$$

with initial value $G(0)=S(0)$. If $\{S(t) \mid t \geq 0\}$ has continuous paths, then the integral in (9) is a path-wise Riemann integral, a.s.

Assume now a discrete-time stock price process, i.e. $I=\left\{t_{0}, t_{1}, t_{2}, \ldots, t_{N}\right\}$, with $t_{0}=0$ and $t_{N}=T$. Moreover, we additionally assume an equidistant time grid, hence $\Delta t=$ $t_{j}-t_{j-1}$ for all $j=1,2, \ldots, N$. The annualized dividend rate is denoted by $\nu$ and assumed to be constant, deterministic and paid out at the end of each period. Define:

$$
\Delta D\left(t_{j}\right)=D\left(t_{j}\right)-D\left(t_{j-1}\right), \text { for } j=1,2, \ldots, N
$$

where $D(t)$ denotes the value of dividends paid in the interval $(0, t)$. Then:

$$
\Delta D\left(t_{j}\right)=\nu S\left(t_{j}\right) \Delta t
$$

The gains process $\{G(t) \mid t \in I\}$ is then defined as follows:

$$
G\left(t_{j}\right)=S\left(t_{j}\right)+\sum_{k=1}^{j} \mathrm{e}^{r\left(t_{j}-t_{k}\right)} \Delta D\left(t_{j}\right),
$$

with initial value $G(0)=S(0)$.

Loosely speaking, the financial market is said to be arbitrage-free if there is no selffinancing trading strategy that does not require the injection of capital at initiation, while it leads to a non-negative terminal gain with probability 1 and to a positive terminal gain with a positive probability. The financial market is said to be complete if any contingent claim admits a replicating strategy. A precise definition of these notions requires a careful specification of the set of admissable investment strategies. For a detailed description of these concepts, we refer e.g. to Karatzas \& Shreve (1998).

Recall that a probability measure $\mathbb{Q}$ defined on $\left(\Omega, \mathcal{F},\left(\mathcal{F}_{t}\right)_{0 \leq t \leq T}\right)$ is said to be an equivalent martingale measure (or a risk-neutral measure) for the financial market described above if $\mathbb{Q}$ is equivalent to $\mathbb{P}$ and any gains process, discounted at the risk-free rate, is a martingale with respect to $\mathbb{Q}$. In particular, under the risk-neutral measure $\mathbb{Q}$ we have that

$$
\begin{aligned}
S(0) & =\mathrm{e}^{-r t} \mathbb{E}[G(t)] \\
& =\mathrm{e}^{-r t} \mathbb{E}[S(t)]+\mathbb{E}\left[\int_{0}^{t} \mathrm{e}^{-r u} \mathrm{~d} D(u)\right] .
\end{aligned}
$$

In this expression and also hereafter, expectations have to be interpreted as expectations under the $\mathbb{Q}$-measure. Furthermore, the notation $F_{S(t)}$ will be used to denote the cdf of the stock price $S(t)$ under $\mathbb{Q}$. 
Remark 5 Consider the r.v.'s $X$ and $Y$, and let $\mathbb{Q}$ be a probability measure, which is equivalent to the underlying probability $\mathbb{P}$. Then it holds:

$$
X=Y \quad \mathbb{P}-\text { a.s. if, and only if, } X=Y \quad \mathbb{Q}-\text { a.s. }
$$

From the First Fundamental Theorem of Asset Pricing we know that the no-arbitrage condition is 'essentially equivalent' to the existence of an equivalent martingale measure $\mathbb{Q}$. For a detailed discussion of this fundamental theorem in mathematical finance, we refer to Delbaen \& Schachermayer (2008). Consider a traded contingent claim $H$ which has a pay-off of $H(S(t))$ at time $t$. If the risk-neutral pricing measure in the market is given by $\mathbb{Q}$, then the price of $H(S(t))$ at time $s$, where $t \geq s \geq 0$, is equal to the conditional expected value of its discounted pay-off at time $t$ :

$$
\text { Price of } H(S(t)) \text { at time } s=\mathrm{e}^{-r(t-s)} \mathbb{E}\left[H(S(t)) \mid \mathcal{F}_{s}\right],
$$

where the expectation is taken with respect to $\mathbb{Q}$. Hereafter, we will assume that there exists a risk-neutral measure $\mathbb{Q}$, and hence that the market is free of arbitrage.

In the sequel of this paper, we always assume that the stock $S$ pays no dividends in $[0, T]$. Our results remain valid in the more general context where some of the stocks are paying dividends, provided the earned dividends are reinvested in the stock.

\section{A market with a single non-dividend paying risky asset}

In this subsection, we consider the financial market defined in Section 3 and with a single non-dividend paying risky asset, a stock let's say. The current price of the stock is given by $S(0)$ and its price process is given by the stochastic process $\{S(t) \mid 0 \leq t \leq T\}$. We assume that the market is arbitrage-free in the sense that prices of contingent claims are determined via the discounted expected pay-off, where expectations are taken under some equivalent martingale measure $\mathbb{Q}$.

Assume that $0 \leq t_{1}<t_{2}<\ldots<t_{n} \leq T$. Let us also assume that the risk-neutral distributions of $S\left(t_{1}\right), S\left(t_{2}\right), \ldots, S\left(t_{n}\right)$ are known. A natural question which arises now is whether an artificial market where the price vector $\left(S\left(t_{1}\right), S\left(t_{2}\right), \ldots, S\left(t_{n}\right)\right)$ is comonotonic is reachable, in the sense that it is arbitrage-free. Assuming that the marginal pricing distributions are fixed, this question can be restated as follows: does there exist an arbitrage-free market model, where the copula of $\left(S\left(t_{1}\right), S\left(t_{2}\right), \ldots, S\left(t_{n}\right)\right)$ in the $\mathbb{Q}$-world is the comonotonic copula?

As the measure $\mathbb{P}$ and any equivalent martingale measure agree on sure events, almost sure relationships in the $\mathbb{P}$-world immediately translate in almost sure relationships in the pricing world and vice versa. Taking into account Definition 2, we can conclude

\footnotetext{
${ }^{1}$ If option prices with maturities $t_{1}, t_{2}, \ldots$ can be observed, the marginal risk-neutral distributions can be determined; see e.g. Breeden \& Litzenberger (1978).
} 
that comonotonicity of $\left(S\left(t_{1}\right), S\left(t_{2}\right), \ldots, S\left(t_{n}\right)\right)$ in the risk-neutral world is equivalent to comonotonicity of this vector in the $\mathbb{P}$-world; see e.g. Dhaene, Kukush, Luciano, Schoutens \& Stassen (2013).

In the following subsection, we derive conditions under which it is possible to have a comonotonic vector $\left(S\left(t_{1}\right), S\left(t_{2}\right), \ldots, S\left(t_{n}\right)\right)$, without violating the no-arbitrage assumption. We have a closer look at the Black \& Scholes model in Example 12.

\subsection{An arbitrage-free and comonotonic market}

In order to be able to answer the question on reachability of the comonotonic market situation, we first have to prove the following lemma.

Lemma 6 Consider the general arbitrage-free financial market with a single non-dividend paying stock and with risk-neutral measure $\mathbb{Q}$. In case there exists a functional relationship between the stock prices $S(s)$ and $S(t)$ at respective dates $s$ and $t$ with $s \leq t$, in the sense that

$$
S(t)=f(S(s))
$$

for some, not necessarily known, function $f$, then this relationship must be linear:

$$
S(t)=S(s) \mathrm{e}^{r(t-s)} .
$$

Proof. We know that for the non-dividend paying stock $S$, we have that

$$
S(s)=\mathrm{e}^{-r(t-s)} \mathbb{E}\left[S(t) \mid \mathcal{F}_{s}\right] .
$$

Taking into account the relation 12 , this expression can be transformed into

$$
S(s)=\mathrm{e}^{-r(t-s)} S(t),
$$

which is equivalent to $(13)$.

Relation 13 is a statement in the $\mathbb{P}$-world. Because $\mathbb{P}$ and $\mathbb{Q}$ are equivalent measures, it also holds in the $\mathbb{Q}$ world.

\subsection{The continuous case}

Lemma 6 allows us to characterize comonotonicity of the vector $\left(S\left(t_{1}\right), S\left(t_{2}\right), \ldots, S\left(t_{n}\right)\right)$ of stock prices in an arbitrage-free market. Notice that $S\left(t_{1}\right)$ has a continuous cdf under $\mathbb{P}$ if, and only if, it has a continuous cdf under $\mathbb{Q}$.

Theorem 7 Consider the general arbitrage-free financial market with a single non-dividendpaying stock and with risk-neutral measure $\mathbb{Q}$. Assuming that $F_{S\left(t_{1}\right)}$ is a continuous cdf, the following statements are equivalent: 
(1)

$$
\left(S\left(t_{1}\right), S\left(t_{2}\right), \ldots, S\left(t_{n}\right)\right) \text { is comonotonic. }
$$

(2) For any couple $\left(S\left(t_{1}\right), S\left(t_{j}\right)\right)$, the following $\mathbb{P}$-a.s. relationship holds:

$$
S\left(t_{j}\right)=S\left(t_{1}\right) \mathrm{e}^{r\left(t_{j}-t_{1}\right)}, \quad j=1,2, \ldots, n .
$$

Proof. (1) Let us first assume that $\left(S\left(t_{1}\right), S\left(t_{2}\right), \ldots, S\left(t_{n}\right)\right)$ is a comonotonic random vector. As $F_{S\left(t_{1}\right)}$ is continuous, it follows from Theorem 4 that there exist non-decreasing functions $f_{i}, i=2,3, \ldots, n$, such that

$$
\left(S\left(t_{1}\right), S\left(t_{2}\right), \ldots, S\left(t_{n}\right)\right)=\left(S\left(t_{1}\right), f_{2}\left(S\left(t_{1}\right)\right) \ldots, f_{n}\left(S\left(t_{1}\right)\right)\right) .
$$

The linear relationships (14) follow then from Lemma 6.

(2) The conditions (14) can be rewritten as

$$
\left(S\left(t_{1}\right), S\left(t_{2}\right), \ldots, S\left(t_{n}\right)\right)=\left(S\left(t_{1}\right), S\left(t_{1}\right) \mathrm{e}^{r\left(t_{2}-t_{1}\right)}, \ldots, S\left(t_{1}\right) \mathrm{e}^{r\left(t_{n}-t_{1}\right)}\right) .
$$

This implies that $\left(S\left(t_{1}\right), S\left(t_{2}\right), \ldots, S\left(t_{n}\right)\right)$ is comonotonic.

In Chen et al. (2015), the authors prove that strict comonotonicity of the price vector $\left(S\left(t_{1}\right), S\left(t_{2}\right), \ldots, S\left(t_{n}\right)\right)$ is equivalent with (14), while Albrecher et al. (2008) prove Theorem 7 for the case where all marginal cdf's $F_{S\left(t_{i}\right)}, i=1,2, \ldots, n$ are continuous. Notice that Theorem 7 is directly applicable to many Lévy market models, as in most of these models $S\left(t_{1}\right)$ has a continuous cdf.

An essential assumption in Theorem 7 is the existence of a risk-neutral pricing measure $\mathbb{Q}$. Notice however, that the theorem remains to hold under the somewhat weaker assumption that the market is arbitrage-free, provided we assume additionaly that the risk-neutral pricing distributions $F_{S\left(t_{i}\right)}, i=1,2, \ldots, n$ of the individual stocks are known. This result follows from Remark ?? and the proof of Theorem 4.

Returning to the question that we considered in the beginning of this section, we can conclude from Theorem 7 that in case the marginal risk-neutral distributions $F_{S\left(t_{i}\right)}, i=$ $1,2, \ldots, n$, are given and $F_{S\left(t_{1}\right)}$ is a continuous cdf, it will in general be impossible to find an arbitrage-free market where the corresponding dependence structure is characterized by the comonotonic copula, unless these marginals cdf's are such that they fulfill the conditions (14). In the following example we show that the equivalence of (1) and (2) in Theorem 7 will not necessarily remain to hold in case $S\left(t_{1}\right)$ has a discrete cdf.

Example 8 Consider the well-known Cox-Ross-Rubinstein binomial model in the time interval $[0,2]$. In this model, there is a bank account with constant interest intensity $r$ and a non-dividend paying stock of which we observe the prices $S(0), S(1)$ and $S(2)$ at respective times $t=0,1,2$. The stock price $S(0)$ at time 0 is given. Moreover, the stock price dynamics follow from

$$
S(t+1)=S(t) \times Z(t+1), \quad t=0,1,
$$


where $Z(1)$ and $Z(2)$ are i.i.d. r.v.'s which can only take positive values $d$ and $u$, with $0<d<u$, such that

$$
\mathbb{P}[Z(t+1)=u]=1-\mathbb{P}[Z(t+1)=d]=p,
$$

for some $0<p<1$. The couple $(S(1), S(2))$ is comonotonic since it has a comonotonic support. A necessary and sufficient condition for this model to be arbitrage-free is that

$$
d<\mathrm{e}^{r}<u
$$

or equivalently, that there exists an equivalent martingale measure $\mathbb{Q}$; see e.g. Björk (1998). The equivalent martingale measure is determined by

$$
\mathbb{Q}[Z(t+1)=u]=1-\mathbb{Q}[Z(t+1)=d]=\frac{\mathrm{e}^{r}-d}{u-d} .
$$

Any choice of $d, u$ and $r$ fulfilling the condition (15) leads to a discrete arbitrage-free market where $(S(1), S(2))$ is comonotonic, although the a.s. linear relationship (14) is violated.

\subsection{A generalization}

From Theorem 7 we find that with given marginals, a comonotonic and arbitrage-free market is in general not attainable when $F_{S\left(t_{1}\right)}$ is continuous. In this section we generalize Theorem 7 by relaxing the assumption that the stock price $S\left(t_{1}\right)$ at time $t_{1}$ has a continuous cdf. We first introduce the notion of an isolated mass point of (the distribution of) a random variable.

Definition 9 A r.v. $X$ is said to have an isolated mass point $x$ under the measure $\mathbb{P}$ if $\mathbb{P}[X=x]>0$ and there exists an $\varepsilon>0$ such that $\mathbb{P}[X \in(x-\varepsilon, x) \cup(x, x+\varepsilon)]=0$.

We say that the r.v. $X$ has no isolated mass under the measure $\mathbb{P}$ if there are no isolated mass points, i.e. if for each $x \in \mathbb{R}$ with $\mathbb{P}[X=x]>0$ and for every $\varepsilon>0$, one has that $\mathbb{P}[X \in(x-\varepsilon, x) \cup(x, x+\varepsilon)]>0$.

Notice that an isolated mass point of $X$ under the measure $\mathbb{P}$ remains an isolated mass point under an equivalent martingale measure, and vice versa. Also, $X$ has no isolated mass under $\mathbb{P}$ if, and only if, it has no isolated mass under any equivalent martingale measure $\mathbb{Q}$.

Theorem 10 Consider the arbitrage-free market with a single non-dividend paying stock. Assume additionally that for any isolated mass point $x$ of $S\left(t_{1}\right)$ and for any $j=2,3 \ldots, n$, we have that

$$
\mathbb{P}\left[S\left(t_{1}\right)=x, S\left(t_{j}\right)<x \mathrm{e}^{r\left(t_{j}-t_{1}\right)}\right] \times \mathbb{P}\left[S\left(t_{1}\right)=x, S\left(t_{j}\right)>x \mathrm{e}^{r\left(t_{j}-t_{1}\right)}\right]=0 .
$$

Then the following statements are equivalent: 


$$
\left(S\left(t_{1}\right), S\left(t_{2}\right), \ldots, S\left(t_{n}\right)\right) \text { is comonotonic. }
$$

(2) For any $j=1,2, \ldots, n$, it holds

$$
S\left(t_{j}\right)=S\left(t_{1}\right) \mathrm{e}^{r\left(t_{j}-t_{1}\right)} .
$$

A proof of Theorem 10 can be found in the appendix. In Example 8, we showed that in the Cox-Ross-Rubinstein binomial model in the time interval $[0,2]$, the vector $\left(S\left(t_{1}\right), S\left(t_{2}\right)\right)$ is comonotonic, without violating the no-arbitrage condition. The set of possible outcomes for $S\left(t_{1}\right)$ is given by $\{S(0) d, S(0) u\}$ and each point in this set is an isolated mass point. However, one can verify that condition (17) is not satisfied, implying that $\left(S\left(t_{1}\right), S\left(t_{2}\right)\right)$ can be comonotonic without having the almost sure relationship (18).

Corollary 1 Consider the arbitrage-free market with a single non-dividend paying stock. Assume additionally that $S\left(t_{1}\right)$ has no isolated mass. Then statements (1) and (2) from Theorem 10 are equivalent.

As a special case from Corollary 1 we find that statements (1) and (2) from Theorem 10 are equivalent in case $S\left(t_{1}\right)$ has a continuous cdf, implying that Theorem 7 is a special case of Theorem 10 .

Example 11 (An arbitrage-free market with comonotonic modification) Let the cdf $F_{S\left(t_{1}\right)}$ be such that there exists an isolated mass point $x$ of $S\left(t_{1}\right)$, and

$$
\begin{aligned}
& p_{1}=\mathbb{P}\left[S\left(t_{2}\right)<x \mathrm{e}^{r\left(t_{2}-t_{1}\right)}\right]>0, \\
& p_{2}=\mathbb{P}\left[S\left(t_{2}\right)>x \mathrm{e}^{r\left(t_{2}-t_{1}\right)}\right]>0 .
\end{aligned}
$$

Then one can construct an arbitrage-free market with comonotonic asset prices $\left(S\left(t_{1}\right), S\left(t_{2}\right)\right)$ such that the almost sure relationship (18) does not hold. Indeed, we have that

$$
q=1-p_{1}-p_{2}=\mathbb{P}\left[S\left(t_{2}\right)=x \mathrm{e}^{r\left(t_{2}-t_{1}\right)}\right] .
$$

Let $m=\mathbb{P}\left[S\left(t_{1}\right)=x\right]$, and define $F_{1}$ and $F_{2}$ as follows:

$$
\begin{aligned}
& F_{1}(y)=\mathbb{P}\left[S\left(t_{1}\right) \leq y \mid S\left(t_{1}\right) \leq x\right], \\
& F_{2}(y)=\mathbb{P}\left[S\left(t_{1}\right) \leq y \mid S\left(t_{1}\right) \geq x\right] .
\end{aligned}
$$

The conditional cdf's $G_{1}$ and $G_{2}$ are defined as

$$
\begin{aligned}
& G_{1}(y)=\mathbb{P}\left[S\left(t_{2}\right) \leq y \mid S\left(t_{2}\right)<x \mathrm{e}^{r\left(t_{2}-t_{1}\right)}\right], \\
& G_{2}(y)=\mathbb{P}\left[S\left(t_{2}\right) \leq y \mid S\left(t_{2}\right)>x \mathrm{e}^{r\left(t_{2}-t_{1}\right)}\right] .
\end{aligned}
$$

Then we construct the cdf of $\underline{Z}$ with

$$
\begin{gathered}
\mathbb{P}\left[\underline{Z}=\left(x, x \mathrm{e}^{r\left(t_{2}-t_{1}\right)}\right)\right]=m q, \\
\mathbb{P}\left[\underline{Z}=\left(F_{1}^{-1}(U), G_{1}^{-1}(U)\right)\right]=p_{1}(1-m q), \\
\mathbb{P}\left[\underline{Z}=\left(F_{2}^{-1}(U), G_{2}^{-1}(U)\right)\right]=p_{2}(1-m q) .
\end{gathered}
$$


The random vector $\underline{Z}$ has marginal distributions identical to the distributions of $S\left(t_{1}\right)$ and $S\left(t_{2}\right)$, respectively. Furthermore, the market with comonotonic asset prices $\left(Z_{1}, Z_{2}\right)$ is arbitrage-free, due to the fact that the probabilities

$$
\mathbb{P}\left[\underline{Z}<\left(x, x \mathrm{e}^{r\left(t_{2}-t_{1}\right)}\right)\right] \text { and } \mathbb{P}\left[\underline{Z}>\left(x, x \mathrm{e}^{r\left(t_{2}-t_{1}\right)}\right)\right]
$$

are positive. We also find that in this comonotonic and arbitrage-free market the almost sure relationship (18) does not hold. Let $\underline{Z}=\left(Z\left(t_{1}\right), Z\left(t_{2}\right)\right)$. We set $Z(t)=Z\left(t_{1}\right) \mathrm{e}^{r\left(t-t_{1}\right)}$ if $0 \leq t<t_{1}$, and $Z(t)=Z\left(t_{2}\right) \mathrm{e}^{r\left(t-t_{2}\right)}$ if $t_{2} \leq t \leq T$. We use the natural filtration generated by the process $Z(t)$, where $\mathbb{P}$-null sets are added to each sigma-field of the filtration. Then the constructed market is arbitrage- free, the random vector $\underline{Z}$ is comonotonic, and the proportionality condition (18) does not hold for $t_{1}$ and $t_{2}$.

In case the stock price process $\{S(t) \mid t \geq 0\}$ can be described by the exponential function of a Lévy process with Brownian part, the cdf $F_{S(t)}$ is continuous for any $t$ and, consequently, comonotonicity of $\left(S\left(t_{1}\right), S\left(t_{2}\right), \ldots, S\left(t_{n}\right)\right)$ is, in general, not attainable in an arbitrage-free market. More details about the use of Lévy processes in finance can be found in Schoutens (2003).

\section{Example 12 (An arbitrage-free market without comonotonic modification)}

Consider a financial market with a constant and deterministic risk-free interest $r \geq 0$ and a single non-dividend paying stock. Suppose that the only information that is available about the random vector $\left(S\left(t_{1}\right), S\left(t_{2}\right), \ldots, S\left(t_{n}\right)\right)$ is that its risk-neutral marginal distributions follow from

$$
\ln \frac{S\left(t_{j}\right)}{S(0)} \stackrel{\mathbb{Q}-d}{=} N\left(\left(r-\frac{1}{2} \sigma^{2}\right) t_{j}, \sigma^{2} t_{j}\right), j=1,2, \ldots, n,
$$

with $\sigma>0$. Here, $\stackrel{\mathbb{Q}-d}{=}$ denotes an equality in distribution under the $\mathbb{Q}$-measure. This information about the market may be observed e.g. via traded European option prices. This information is feasible in the sense that there exists an arbitrage-free market model where the risk-neutral distributions of the $S\left(t_{j}\right)$ are given by (19). Indeed, consider the Black \& Scholes model where under $\mathbb{P}$ on has that

$$
\frac{\mathrm{d} S(t)}{S(t)}=\mu \mathrm{d} t+\sigma \mathrm{d} B(t), \text { for } t \geq 0,
$$

where $\mu \geq 0$ and $\{B(t) \mid t \geq 0\}$ is a standard Brownian motion defined on the filtered probability space $(\Omega, \mathcal{F}, \mathbb{P})$. This probability space is equiped with the filtration $\left(\mathcal{F}_{t}\right)_{0 \leq t \leq T}$ of $\mathcal{F}$ which records the 'past behavior' of the multivariate Brownian motion. This means that for $0 \leq t \leq T, \mathcal{F}_{t}$ denotes the $\sigma$-algebra generated by $\{B(s) \mid 0 \leq s \leq t\}$, which we also denote by $\sigma\{B(s) \mid 0 \leq s \leq t\}$, completed by events of zero $\mathbb{P}$-probability. It is well-known that the Black \& Scholes model is arbitrage free and complete. Under the unique martingale measure $\mathbb{Q}$, we have that

$$
\ln \frac{S(t)}{S(0)} \stackrel{\mathbb{Q}-d}{=} N\left(\left(r-\frac{1}{2} \sigma^{2}\right) t, \sigma^{2} t\right) .
$$


This implies that the risk-neutral distribtutions of $\ln \frac{S\left(t_{j}\right)}{S(0)}$ are indeed given by 19 .

One can easily verify that in case the risk-neutral distributions $F_{S\left(t_{i}\right)}$ follow from $(19)$, then for any $j \geq 2$, it holds that

$$
S\left(t_{j}\right) \stackrel{\mathbb{Q}-d}{\neq} S\left(t_{1}\right) \mathrm{e}^{r\left(t_{j}-t_{1}\right)}, \quad j=2, \ldots, n,
$$

where the notation $\stackrel{\mathbb{Q}-d}{\neq}$ is used to indicate that $S\left(t_{j}\right)$ and $S\left(t_{1}\right) \mathrm{e}^{r\left(t_{j}-t_{1}\right)}$ have different riskneutral distributions. The inequalities (20) immediately imply that (14) does not hold. From Theorem 7 we can conclude that in case the risk-neutral distributions of the stock prices $S\left(t_{j}\right)$ follow from $(19)$, then it is impossible to find an arbitrage-free market where the vector $\left(S\left(t_{1}\right), S\left(t_{2}\right), \ldots, S\left(t_{n}\right)\right)$ is comonotonic.

\section{A multiple asset market}

In this section, we consider a financial market consisting of $n$ stocks, labeled from 1 to $n$, and a bank account. The individual stocks can be described as in Section 3. The stocks may be dividend-paying or not. The prices of these stocks at a fixed future time $T$ are given by the r.v.'s $S_{i}(T), i=1,2, \ldots, n$. Furthermore, the market is assumed to be arbitrage-free in the sense that prices are determined via some equivalent martingale measure $\mathbb{Q}$. In order to avoid unnecessary overloading of the notations, hereafter we will omit the fixed time index $T$ when no confusion is possible. Hence, the random vector $\left(S_{1}(T), S_{2}(T), \ldots, S_{n}(T)\right)$ will be denoted as $\left(S_{1}, S_{2}, \ldots, S_{n}\right)$ if no confusion is possible.

Assume the only information available about the random behavior of the future prices of the different assets at a fixed time $T$ is the set of marginal risk-neutral pricing distributions $F_{S_{i}}, i=1,2, \ldots, n$. The question arises whether this limited information always allows for a market where the vector $\left(S_{1}, S_{2}, \ldots, S_{n}\right)$ is comonotonic. Notice that comonotonicity under $\mathbb{P}$ is equivalent to comonotonicity under the equivalent martingale measure. The answer to our question is 'yes'. Indeed, if we set

$$
S_{i}=F_{S_{i}}^{-1}(U), i=1,2, \ldots, n,
$$

where $U \sim$ Uniform $(0,1)$ and

$$
S_{i}(0)=\mathrm{e}^{-r T} \mathbb{E}\left[S_{i}\right], i=1,2, \ldots, n,
$$

then the market with discrete times $t=0, T$ and asset prices $S_{i}(t), i=1,2, \ldots, n$ is comonotonic and has marginal cdf's $F_{S_{i}}, i=1,2$. A proof of this result is also given in Theorem 3.1 of Hobson et al. (2005). A related result can also be found in Proposition 3.6 of Tavin (2012).

\subsection{The multivariate Black \& Scholes model}

Consider the financial market described in the previous section and assume that the price dynamics of stock $i$ under $\mathbb{P}$ follow from

$$
\frac{\mathrm{d} S_{i}(t)}{S_{i}(t)} \stackrel{\mathbb{P}}{=} \mu_{i} \mathrm{~d} t+\sigma_{i} \mathrm{~d} B_{i}(t), \quad t \geq 0 \text { and } i=1,2, \ldots, n
$$


where $\mu_{i} \geq 0$ is the drift and $\sigma_{i}>0$ the volatility of stock $i$. The parameters $r, \mu_{i}$ and $\sigma_{i}$ are assumed to be deterministic and constant over time. Furthermore, $\{\underline{B}(t) \mid t \geq 0\}$ is an $n$-dimensional correlated Brownian motion process defined on the probability space $(\Omega, \mathcal{F}, \mathbb{P})$. This means that the stochastic processes $\left\{B_{i}(t) \mid t \geq 0\right\}$ are standard Brownian motions and the dependence structure of $\{\underline{B}(t) \mid t \geq 0\}$ is captured by

$$
\operatorname{Cov}\left[\sigma_{i} B_{i}(t), \sigma_{j} B_{j}(t+s)\right]=\sigma_{i j} t, \text { for any } t, s \geq 0,
$$

with parameters $\sigma_{i j}, i, j=1,2, \ldots, n$, which are also assumed to be deterministic and constant over time.

The probability space $(\Omega, \mathcal{F}, \mathbb{P})$ is equipped with the natural filtration $\left(\mathcal{F}_{t}\right)_{0 \leq t \leq T}$ of $\mathcal{F}$, which records the 'past behavior' of the multivariate Brownian motion process. The set $\mathcal{F}_{t}$ is the $\sigma$-algebra $\sigma\{\underline{B}(s), s \leq t\}$ completed by events of zero probability. In the sequel, we will refer to this model as the Multivariate Black 8 Scholes model.

Hereafter, the notation $\underline{1}$ is used for the $n \times 1$ matrix with all entries equal to 1 . Furthermore, we denote the $n \times 1$ matrix of the drifts by $\underline{\mu}$, while the $n \times n$ matrix with entries $\sigma_{i j}$ is denoted by $\underline{\Sigma}$. The range $R(\underline{\Sigma})$ of $\underline{\Sigma}$ is defined by

$$
R(\underline{\Sigma})=\{\underline{y} \mid \underline{y}=\underline{\Sigma} \underline{x} \text { for some } n \times 1-\operatorname{matrix} \underline{x}\} .
$$

The Black \& Scholes model (21) is not necessarily arbitrage-free. A precise definition of the notions of arbitrage and completeness within the Black \& Scholes model is considered e.g. in Dhaene, Kukush \& Linders (2013) and the references therein. Concerning the absence of arbitrage in the Black \& Scholes model (21), the following result holds.

Theorem 13 In the Black 85 Scholes model (21), the following statements are equivalent:

(1) The model is arbitrage-free.

(2) There exists an equivalent martingale measure.

(3) There exists a unique equivalent martingale measure.

(4) $\underline{\mu}-r \underline{1} \in R(\underline{\Sigma})$.

When the Black \& Scholes model (21) is arbitrage-free, the risk-neutral pricing dynamics of the stocks follows from

$$
\frac{\mathrm{d} S_{i}(t)}{S_{i}(t)} \stackrel{\mathbb{Q}}{=} r \mathrm{~d} t+\sigma_{i} \mathrm{~d} B_{i}(t), \quad i=1,2, \ldots, n .
$$

In particular, we find that in the $\mathbb{Q}$-world, $\ln \frac{S_{i}(t)}{S_{i}(0)}$ follows a normal distribution with mean $\left(r-\frac{1}{2} \sigma_{i}^{2}\right) t$ and standard deviation $\sigma_{i} \sqrt{t}$. Hence we have that

$$
\ln \frac{S_{i}(t)}{S_{i}(0)} \stackrel{\mathbb{Q}-d}{=} N\left(\left(r_{i}-\frac{1}{2} \sigma_{i}^{2}\right) t, \sigma_{i}^{2} t\right) .
$$

As the matrix $\underline{\Sigma}$ is necessary positive semidefinite, we have that $\underline{\Sigma}$ is positive definite if and only if $R(\underline{\Sigma})=\mathbb{R}^{n}$. Combining this observation with Theorem 13 , we find the following corollary. 
Corollary 2 A sufficient (but not necessary) condition for the Black \& Scholes model (21) to be arbitrage-free is that $\underline{\Sigma}$ is positive definite.

Hereafter, we consider some special cases of the Black \& Scholes model (21). We will say that the Black \& Scholes market is comonotonic if the stock price vector $\left(S_{1}, S_{2}, \ldots, S_{n}\right)$ is comonotonic.

Example 14 (a comonotonic B\&S market which is not arbitrage-free) Consider a two dimensional Black \& Scholes model (21)

$$
\frac{\mathrm{d} S_{i}(t)}{S_{i}(t)} \stackrel{\mathbb{P}}{=} \mu_{i} \mathrm{~d} t+\sigma \mathrm{d} B(t), \quad i=1,2 .
$$

From expression (30) in Example 16 , we find that this model can only be arbitrage-free if

$$
\mu_{1}=\mu_{2} .
$$

Assume for the moment that $\mu_{1}>\mu_{2}$ and $S_{1}(0)=S_{2}(0)$. Consider the strategy of buying one unit of the first stock and sell one unit of the second stock. This position does not require any capital input at time $t=0$. However, because the first asset will grow with a faster speed than the second one, we have that

$$
S_{1}(t)>S_{2}(t), \text { for } t>0,
$$

which means that the investor will make a sure profit, with an initial investment of 0 . Here, the B\&S market is comonotonic, but not arbitrage free.

Example 15 (an arbitrage-free B\&S market which is not comonotonic) Consider the following special case of the Black \& Scholes model (21):

$$
\frac{\mathrm{d} S_{i}(t)}{S_{i}(t)} \stackrel{\mathbb{P}}{=} \mu_{i} \mathrm{~d} t+\sigma \mathrm{d} B_{i}(t), \quad i=1,2, \ldots, n,
$$

where $\operatorname{Cov}\left[B_{i}(1), B_{j}(1)\right]<1$. Then, there are two separate Brownian motions driving the market and hence this market is not comonotonic.

In this case we find that

$$
\sigma_{i j}=\sigma^{2} \operatorname{Cov}\left[B_{i}(1), B_{j}(1)\right] .
$$

Let us now assume that the matrix $\underline{\Sigma}$ is positive definite. From our considerations above, we find that the model (26) is arbitrage-free and complete. Its risk-neutral pricing dynamics follows from

$$
\frac{\mathrm{d} S_{i}(t)}{S_{i}(t)} \stackrel{\mathbb{Q}}{=} r t+\sigma \mathrm{d} B_{i}(t), \quad i=1,2, \ldots, n .
$$

In particular, we find that

$$
\ln \frac{S_{i}(t)}{S_{i}(0)} \stackrel{\mathbb{Q}-d}{=} N\left(\left(r-\frac{1}{2} \sigma^{2}\right) t, \sigma^{2} t\right) .
$$


We can conclude that we have found an arbitrage-free B\&S market, where the price vector $\left(S_{1}, S_{2}, \ldots, S_{n}\right)$ is not comonotonic.

Example 16 (arbitrage-free and comonotonic B\&S market) Consider the following special case of the Black \& Scholes model (21):

$$
\frac{\mathrm{d} S_{i}(t)}{S_{i}(t)} \stackrel{\mathbb{P}}{=} \mu_{i} \mathrm{~d} t+\sigma_{i} \mathrm{~d} B(t), \quad i=1,2, \ldots, n,
$$

where the stochasticity of all stock prices is captured by a single standard Brownian motion process $\{B(t) \mid t \geq 0\}$. In this model, we have that for each time $t$, the price vector $\left(S_{1}(t), \ldots, S_{n}(t)\right)$ is comonotonic because each $S_{i}(t)$ is an increasing function of the same r.v. $B(t)$. The process $\{B(t) \mid t \geq 0\}$ plays the role of a random index which influences the behavior of stock prices in the market. In this case we find that

$$
\sigma_{i j}=\sigma_{i} \sigma_{j} .
$$

The range of $\underline{\Sigma}$ is given by

$$
R(\underline{\Sigma})=\left\{\left(\sum_{j=1}^{n} \sigma_{j} x_{j}\right)\left(\begin{array}{c}
\sigma_{1} \\
\sigma_{2} \\
\vdots \\
\sigma_{n}
\end{array}\right) \mid \underline{x} \in \mathbb{R}^{n}\right\} .
$$

Taking into account that $\left\{\left(\sum_{j=1}^{n} \sigma_{j} x_{j}\right) \mid \underline{x} \in \mathbb{R}^{n}\right\}=\mathbb{R}$, we can rewrite the previous expression as

$$
R(\underline{\Sigma})=\left\{\lambda\left(\begin{array}{c}
\sigma_{1} \\
\sigma_{2} \\
\vdots \\
\sigma_{n}
\end{array}\right) \mid \lambda \in \mathbb{R}\right\} .
$$

From Theorem 13 we find that the Black \& Scholes model (21) is arbitrage-free if, and only if, there exists a common real number $c$ such that

$$
\frac{\mu_{i}-r}{\sigma_{i}}=c, \quad i=1,2, \ldots, n .
$$

The risk-neutral price dynamics of the model 29p follow from:

$$
\frac{\mathrm{d} S_{i}(t)}{S_{i}(t)} \stackrel{\mathbb{Q}}{=} r \mathrm{~d} t+\sigma_{i} \mathrm{~d} B(t), \quad i=1,2, \ldots, n .
$$

In particular, we find that

$$
\ln \frac{S_{i}(t)}{S_{i}(0)} \stackrel{\mathbb{Q}-d}{=} N\left(\left(r-\frac{1}{2} \sigma_{i}^{2}\right) t, \sigma_{i}^{2} t\right) .
$$


We can conclude that under the condition (30), the B\&S market is comonotonic and arbitrage-free.

Until here, we assumed that the risk-neutral pricing distributions of the individual stocks are given. Let us now consider the situation where the real-world pricing distributions of the individual stocks are given. In the following example, we show that in this case it is not always possible to find an arbitrage-free market with comonotonic price vector $\left(S_{1}, S_{2}, \ldots, S_{n}\right)$.

Example 17 (comonotonic B\&S market which is not arbitrage-free) Suppose that the real-world distributions of the price at time $T$ of the $n$ assets follow from

$$
\ln \frac{S_{i}}{S_{i}(0)} \stackrel{\mathbb{P}-d}{=} N\left(\left(\mu_{i}-\frac{1}{2} \sigma^{2}\right) T, \sigma^{2} T\right), \quad i=1,2, \ldots, n,
$$

with

$$
\mu_{1}<\mu_{2}<\ldots<\mu_{n}
$$

Here, we write $\stackrel{\mathbb{P}-d}{=}$ to denote an equality in distribution under the measure $\mathbb{P}$. These marginal distributions may be observed in the sense that they are equal to the real world pricing distributions of the arbitrage-free Black \& Scholes model that we considered in Example 15.

In this case it is impossible to construct an arbitrage-free market model where the random vector $\left(S_{1}, S_{2}, \ldots, S_{n}\right)$ is comonotonic. Indeed, in case this vector was comonotonic, we would find that

$$
\left(S_{1}, S_{2}, \ldots, S_{n}\right) \stackrel{\mathbb{P}-d}{=}\left(S_{1}(0) \mathrm{e}^{\left(\mu_{1}-\frac{1}{2} \sigma^{2}\right) T+\sigma \sqrt{T} \Phi^{-1}(U)}, \ldots, S_{n}(0) \mathrm{e}^{\left(\mu_{n}-\frac{1}{2} \sigma^{2}\right) T+\sigma \sqrt{T} \Phi^{-1}(U)}\right)
$$

which holds in the real world. An arbitrage opportunity arises by buying e.g. $\frac{S_{1}(0)}{S_{2}(0)}$ units of the the second asset and selling one unit of the first asset at time 0, while closing this position at time $T$.

\section{Conclusions}

In this note we investigated the relationship between arbitrage-free markets and comonotonicity of traded asset prices. We first investigated the meaning of comonotonicity of the prices $\left(S\left(t_{1}\right), \ldots, S\left(t_{n}\right)\right)$ of a single asset at consecutive dates $t_{1}, t_{2}, \ldots, t_{n}$ in an arbitragefree market with a single risky asset paying a continuous dividend yield $\nu$ and a risk-free bank account with interest intensity $r$. We proved that the vector of subsequent prices $\left(S\left(t_{1}\right), \ldots, S\left(t_{n}\right)\right)$ is comonotonic only under the a.s. linear relationship

$$
S\left(t_{j}\right)=S\left(t_{i}\right) \mathrm{e}^{(r-\nu)\left(t_{j}-t_{i}\right)}, i, j=1,2, \ldots, n
$$


provided $S\left(t_{1}\right)$ has a continuous cdf, or more generally, provided the distribution of $S\left(t_{1}\right)$ has no isolated mass.

Next, we considered an arbitrage-free financial market consisting of $n$ traded assets and a risk-free bank account. The prices of these assets at a fixed future time $T$ are given by the r.v.'s $S_{i}(T), i=1,2, \ldots, n$. We considered situations under which the risk-neutral pricing distributions (or the real world distributions) of the $S_{i}(T)$ are given and investigated whether there exists an arbitrage-free model with the same marginal distributions, under which the asset price vector $\left(S_{1}(T), S_{2}(T), \ldots, S_{n}(T)\right)$ is comonotonic.

Acknowledgement 1 The authors acknowledge the financial support of the Onderzoeksfonds KU Leuven (GOA/13/002: Management of Financial and Actuarial Risks: Modeling, Regulation, Incentives and Market Effects) and the Research Fund Flanders (FWO G.OC38.17N: Measuring diversity and systematic risk from derivative market data and mapping to real-world probability statements). They are grateful to Prof. Yuliya Mishura (Ukraine) and Dr. Xinliang Chen (Belgium) for fruitful discussions.

\section{References}

Albrecher, H., Dhaene, J., Goovaerts, M. \& Schoutens, W. (2005), 'Static hedging of Asian options under Lévy models: the comonotonicity approach', Journal of Derivatives $\mathbf{1 2}(3), 63-72$.

Albrecher, H., Mayer, P. A. \& Schoutens, W. (2008), 'General lower bounds for arithmetic asian option prices', Applied Mathematical Finance 15(2), 123-149.

Barrieu, P. M. \& Scandolo, G. (2013), Assessing financial model risk, Technical report. Available at SSRN: http://ssrn.com/abstract=2284101.

Breeden, D. T. \& Litzenberger, R. H. (1978), 'Prices of state-contingent claims implicit in option prices', Journal of Business 51(4), 621-51.

Chen, X., Deelstra, G., Dhaene, J., Linders, D. \& Vanmaele, M. (2015), 'On an optimization problem related to static super-replicating strategies', Journal of Computational and Applied Mathematics 278, 213-230.

Chen, X., Deelstra, G., Dhaene, J. \& Vanmaele, M. (2008), 'Static super-replicating strategies for a class of exotic options', Insurance: Mathematics \& Economics 42(3), 1067-1085.

Deelstra, G., Dhaene, J. \& Vanmaele, M. (2011), An overview of comonotonicity and its applications in finance and insurance, in B. Oksendal \& G. Nunno, eds, 'Advanced Mathematical Methods for Finance', Springer Berlin Heidelberg, pp. 155-179.

Deelstra, G., Diallo, I. \& Vanmaele, M. (2008), 'Bounds for Asian basket options', J. Comput. Appl. Math. 218, 215-228. 
Deelstra, G., Liinev, J. \& Vanmaele, M. (2004), 'Pricing of arithmetic basket options by conditioning', Insurance: Mathematics and Economics 34(1), 55-77.

Delbaen, F. \& Schachermayer, W. (2008), The mathematics of arbitrage, Springer. correcter second printing, pp. 373.

Denneberg, D. (1997), Non-Additive measure and integral, 2nd edition, Kluwer Academic Publushers. pp. 184.

Dhaene, J., Denuit, M., Goovaerts, M., Kaas, R. \& Vyncke, D. (2002a), 'The concept of comonotonicity in actuarial science and finance: theory', Insurance: Mathematics $\&$ Economics 31(1), 3-33.

Dhaene, J., Denuit, M., Goovaerts, M., Kaas, R. \& Vyncke, D. (2002b), 'The concept of comonotonicity in actuarial science and finance: applications', Insurance: Mathematics E Economics 31(2), 133-161.

Dhaene, J., Dony, J., Forys, M. B., Linders, D. \& Schoutens, W. (2012), Fix - the fear index: Measuring market fear, in 'Topics in Numerical Methods for Finance, Cummins M. et al. (eds.). Springer Proceedings in Mathematics and Statistics'.

Dhaene, J., Kukush, A. \& Linders, D. (2013), 'The multivariate Black \& Scholes market: conditions for completeness and no-arbitrage', Theory of Probability and Mathematical Statistics 88, 1-14.

Dhaene, J., Kukush, A., Luciano, E., Schoutens, W. \& Stassen, B. (2013), 'On the (in-)dependence between financial and actuarial risks', Insurance: Mathematics and Economics 52(3), 522 - 531.

Dhaene, J., Linders, D., Schoutens, W. \& Vyncke, D. (2012), 'The herd behavior index: A new measure for the implied degree of co-movement in stock markets', Insurance: Mathematics and Economics 50(3), 357-370.

Dhaene, J., Linders, D., Schoutens, W. \& Vyncke, D. (2014), 'A multivariate dependence measure for aggregating risks', Journal of Computational and Applied Mathematics $\mathbf{2 6 3}(0), 78-87$.

Hobson, D., Laurence, P. \& Wang, T. (2005), 'Static-arbitrage upper bounds for the prices of basket options', Quantitative Finance 5(4), 329-342.

Kaas, R., Dhaene, J. \& Goovaerts, M. J. (2000), 'Upper and lower bounds for sums of random variables', Insurance: Mathematics and Economics 27(2), 151-168.

Karatzas, I. \& Shreve, S. E. (1998), Methods of Mathematical Finance, Springer-Verlag, New York.

Kerkhof, J., Melenberg, B. \& Schumacher, H. (2010), 'Model risk and capital reserves', Journal of Banking \& Finance 34(1), 267 - 279. 
Linders, D., Dhaene, J., Hounnon, H. \& Vanmaele, M. (2012), Index options: a model-free approach, Research report AFI-1265 FEB, Leuven: KU Leuven - Faculty of Business and Economics.

Linders, D. \& Stassen, B. (2016), 'The multivariate Variance Gamma model: basket option pricing and calibration', Quantitative Finance 16(4), 555-572.

Schoutens, W. (2003), Lévy Processes in Finance: Pricing Financial Derivatives, Wiley.

Simon, S., Goovaerts, M. \& Dhaene, J. (2000), 'An easy computable upper bound for the price of an arithmetic Asian option', Insurance: Mathematics $\&$ Economics 26(2), 175183.

Tavin, B. (2012), Detection of arbitrage in a market with multi-asset derivatives and known risk-neutral marginals, Technical report, Université Paris I Panthéon-Sorbonne. Available at SSRN: http://ssrn.com/abstract $=2117004$.

\section{Appendix: A.1. Proof of Theorem 10}

Proof. We give a proof for the case where $n=2$. The proof for the case $n>2$ is straightforward.

The proof of $(2) \Rightarrow(1)$ is trivial.

Let us now prove $(1) \Rightarrow(2)$.

Assume that (18) does not hold. Let $x_{i}, i \geq 1$, be all the isolated mass points of the r.v. $S\left(t_{1}\right)$. Note that the number of isolated mass points is at most countable. Let $y_{j}, j \geq 1$, be all the other points where $F_{S\left(t_{1}\right)}$ is discontinuous. We introduce the following notations:

$$
\begin{aligned}
& \Omega_{d}^{1}=\left\{\omega \in \Omega \mid S\left(t_{1}\right)(\omega) \in\left\{x_{i}, i \geq 1\right\}\right\}, \\
& \Omega_{d}^{2}=\left\{\omega \in \Omega \mid S\left(t_{1}\right)(\omega) \in\left\{y_{j}, j \geq 1\right\}\right\}, \\
& \Omega_{c}=\Omega \backslash\left(\Omega_{d}^{1} \cup \Omega_{d}^{2}\right) .
\end{aligned}
$$

1. In a first step, we prove that

$$
S\left(t_{2}\right)(\omega)=S\left(t_{1}\right)(\omega) e^{r\left(t_{2}-t_{1}\right)}, \quad \text { for all } \omega \in \Omega_{c} \text {, a.s. }
$$

Here we suppose that $\mathbb{P}\left[\Omega_{c}\right]>0$ (otherwise it is clear that A1 holds true).

Define the restricted probability space $\left(\Omega_{c}, \mathcal{F} \cap \Omega_{c}, \mathbb{P} / \mathbb{P}\left(\Omega_{c}\right)\right)$ and restricted r.v.'s

$$
\begin{aligned}
S^{r}\left(t_{i}\right)(\omega) & =S\left(t_{i}\right)(\omega), \quad \omega \in \Omega_{c}, i=1,2, \\
S^{r}(0) & =S(0) .
\end{aligned}
$$


Then $S^{r}\left(t_{1}\right)$ has a continuous cdf, the vector $\left(S^{r}\left(t_{1}\right), S^{r}\left(t_{2}\right)\right)$ is still comonotonic, and the market with a single risky stock $S^{r}(t), t=0, t_{1}, t_{2}$, is still arbitrage-free. Then, by Theorem 7, we have that

$$
S^{r}\left(t_{2}\right)(\omega) \stackrel{\text { a.s. }}{=} S^{r}\left(t_{1}\right)(\omega) e^{r\left(t_{2}-t_{1}\right)},
$$

and (A1) follows.

2. Let us now additionally assume that there exists an $i$ such that

$$
\mathbb{P}\left[S\left(t_{1}\right)=x_{i}, S\left(t_{2}\right)>x_{i} \mathrm{e}^{r\left(t_{2}-t_{1}\right)}\right]>0 .
$$

Because $x_{i}$ is an isolated mass point of $S\left(t_{1}\right)$ and (17) holds with $S=x_{i}$, we have that

$$
\mathbb{P}\left[S\left(t_{1}\right)=x_{i}, S\left(t_{2}\right)<x_{i} \mathrm{e}^{r\left(t_{2}-t_{1}\right)}\right]=0 .
$$

Let us now consider the following strategy. At the moment $t_{1}$ the investor observes $S\left(t_{1}\right)$. If $S\left(t_{1}\right) \neq x_{i}$, he does nothing, if $S\left(t_{1}\right)=x_{i}$, he recognizes the situation A2, A3), borrows the amount $x_{i}$ from the bank, immediately buys one unit of the stock and at the moment $t_{2}$ he sells it, returns his debt, and receives $S\left(t_{2}\right)-x_{i} \mathrm{e}^{r\left(t_{2}-t_{1}\right)}$. Overall, $S\left(t_{2}\right)-x_{i} \mathrm{e}^{r\left(t_{2}-t_{1}\right)} \geq 0$, a.s., and $\left.\mathbb{P}\left[S\left(t_{2}\right)-x_{i} \mathrm{e}^{r\left(t_{2}-t_{1}\right)}\right)>0\right]>0$, and we have an arbitrage strategy. This contradicts the condition that the market is arbitrage-free.

3. In a similar way, an arbitrage strategy can be constructed under the condition that there exists an $x_{i}$ with $\mathbb{P}\left[S\left(t_{1}\right)=x_{i}, S\left(t_{2}\right)<x_{i} \mathrm{e}^{r\left(t_{2}-t_{1}\right)}\right]>0$.

4. Thus, from steps 2 and 3 we can conclude that

$$
S\left(t_{2}\right)(\omega)=S\left(t_{1}\right)(\omega) \mathrm{e}^{r\left(t_{2}-t_{1}\right)}, \text { for all } \omega \in \Omega_{d}^{1}, \text { a.s. }
$$

5. Now, suppose that (A1) and (A4) hold and there exists $j$ with

$$
\begin{aligned}
& \mathbb{P}\left[S\left(t_{1}\right)=y_{j}, S\left(t_{2}\right)>y_{j} \mathrm{e}^{r\left(t_{2}-t_{1}\right)}\right]>0, \\
& \mathbb{P}\left[S\left(t_{1}\right)=y_{j}, S\left(t_{2}\right)<y_{j} \mathrm{e}^{r\left(t_{2}-t_{1}\right)}\right]>0 .
\end{aligned}
$$

Then there exists an $\varepsilon \in\left(0, y_{j}\right)$ with

$$
\begin{aligned}
& \mathbb{P}\left[S\left(t_{1}\right)=y_{j}, S\left(t_{2}\right)>\left(y_{j}+\varepsilon\right) \mathrm{e}^{r\left(t_{2}-t_{1}\right)}\right]>0, \\
& \mathbb{P}\left[S\left(t_{1}\right)=y_{j}, S\left(t_{2}\right)<\left(y_{j}-\varepsilon\right) \mathrm{e}^{r\left(t_{2}-t_{1}\right)}\right]>0 .
\end{aligned}
$$

Because $y_{j}$ is not an isolated mass point of $S\left(t_{1}\right)$, we can assume that

$$
\mathbb{P}\left[S\left(t_{1}\right) \in\left(y_{j}, y_{j}+\varepsilon\right)\right]>0 .
$$

(We will exploit (A7), and in case $\mathbb{P}\left[S\left(t_{1}\right) \in\left(y_{j}-\varepsilon, y_{j}\right)\right]>0$ we could exploit (A8)). Now, (A7) and condition (1) of Theorem 10 imply that

$$
\mathbb{P}\left[S\left(t_{1}\right) \in\left(y_{j}, y_{j}+\varepsilon\right), S\left(t_{2}\right)>\left(y_{j}+\varepsilon\right) \mathrm{e}^{r\left(t_{2}-t_{1}\right)}\right]>0,
$$




$$
\mathbb{P}\left[S\left(t_{1}\right) \in\left(y_{j}, y_{j}+\varepsilon\right), S\left(t_{2}\right) \leq\left(y_{j}+\varepsilon\right) \mathrm{e}^{r\left(t_{2}-t_{1}\right)}\right]=0 .
$$

Then due to (A1), there exists $z \in\left(y_{j}, y_{j}+\varepsilon\right)$ which satisfies

$$
\begin{aligned}
& \mathbb{P}\left[S\left(t_{1}\right)=z, S\left(t_{2}\right)>z \mathrm{e}^{r\left(t_{2}-t_{1}\right)}\right]>0, \\
& \mathbb{P}\left[S\left(t_{1}\right)=z, S\left(t_{2}\right)<z \mathrm{e}^{r\left(t_{2}-t_{1}\right)}\right]=0 .
\end{aligned}
$$

These relations are similar to $(\mathrm{A} 2 \mathrm{a}$ ) and $(\mathrm{A} 3 \mathrm{n}$, and part 2 of the proof shows that we get a contradiction.

6. For a fixed $j$, the case A5 with $\mathbb{P}\left[S\left(t_{1}\right)=y_{j}, S\left(t_{2}\right)<y_{j} \mathrm{e}^{r\left(t_{2}-t_{1}\right)}\right]=0$ is impossible due to the reasoning from part 2 of the proof. In a similar way the case (A6) with $\mathbb{P}\left[S\left(t_{1}\right)=y_{j}, S\left(t_{2}\right)>y_{j} \mathrm{e}^{r\left(t_{2}-t_{1}\right)}\right]=0$ is impossible as well.

7. Suppose that (A1) and (A4) hold. Then parts 5 and 6 of the proof imply that $S\left(t_{2}\right)(\omega)=S\left(t_{1}\right)(\omega) \mathrm{e}^{r\left(t_{2}-t_{1}\right)}$, for all $\omega \in \Omega_{d}^{2}$, a.s. Finally, $\left.\left.\mathrm{A} 1\right), \mathrm{A} 4\right)$ and the latter relation imply (18). 